\title{
Prevalência de endoparasitas em codornas japonesas (coturnix coturnix japonica) em dois aviários comerciais na região amazônica
}

\author{
Prevalence of endoparasites in Japanese quail (coturnix coturnix japonica) in two commercial \\ aviaries in the Amazon region \\ Prevalencia de endoparásitos en codornices japonesas (coturnix coturnix japonica) en dos aviarios \\ comerciales de la región amazónica
}

Recebido: 06/07/2021 | Revisado: 12/07/2021 | Aceito: 20/07/2021 | Publicado: 28/07/2021

\author{
Thiago Vaz Lopes \\ ORCID: https://orcid.org/0000-0002-3192-1908 \\ Universidade Federal do Acre, Brasil \\ E-mail: Thiagovlopes@hotmail.com \\ Alexia Souza Maia \\ ORCID: https://orcid.org/0000-0002-3648-7784 \\ Centro Universitário Aparício Carvalho, Brasil \\ E-mail: alexiapvh99@gmail.com \\ Thaíse Letícia Oliveira de Araujo \\ ORCID: https://orcid.org/0000-0002-3091-663X \\ Centro Universitário Aparício Carvalho, Brasil \\ E-mail: thaise1299@gmail.com \\ João Gustavo da Silva Garcia de Souza \\ ORCID: https://orcid.org/0000-0002-5899-2490 \\ Centro Universitário Aparício Carvalho, Brasil \\ E-mail: joaogarciadesouza27@gmail.com \\ Sandro de Vargas Schons \\ ORCID: https://orcid.org/0000-0001-9811-5356 \\ Universidade Federal de Rondônia, Brasil \\ E-mail: sandroschons@unir.br \\ Fernando Andrade Souza \\ ORCID: https://orcid.org/0000-0002-9474-9404 \\ Universidade Federal do Paraná, Brasil \\ E-mail: femedvet@yahoo.com.br
}

\begin{abstract}
Resumo
A área de coturnicultura é uma das que mais se expande, apresentando-se como importante alternativa econômica, sendo o Brasil o quinto maior produtor mundial. Objetivou-se avaliar o perfil endoparasitario que acometem codornas de duas granjas na cidade de Porto Velho - RO. A pesquisa foi realizada em duas granjas de codornas poedeiras na cidade, nos meses de agosto a novembro de 2019. Foram colidas 25 amostras de cada criatório, totalizando 50 amostras, processadas pelo método de WILLIS-MOLLAY para identificação qualitativa dos endoparasitas. Foram positivas todas as 25 amostras (100\%) do G1, sendo possível a identificação dos parasitas dos gêneros Echinuria spp., Trichostrongylus spp., Amidostomum spp., Heterakis gallinarum, Choanotaenia infundibulum, e oocistos de Eimeria spp. No segundo criatório 15 amostras (60\%) apresentavam endoparasitas, entre eles Ascaris spp, Giardia spp., Trichostrongylus spp., com maior prevalência de Amidostomum spp. A exposição desta fauna parasitária em codornas demonstra a importância das pesquisas na coturnicultura e seu potencial desenvolvimento.
\end{abstract}

Palavras- chave: Coturnicultura; Parasitoses; Patologia; Biosseguridade.

\begin{abstract}
The cotton growing area is one of the most expanding, presenting itself as an important economic alternative, with Brazil being the fifth largest producer in the world. The objective was to evaluate the endoparasitic profile that affect quails from two farms in the city of Porto Velho - RO. The research was carried out in two laying quail farms in the city, from August to November 2019. Twenty-five samples were collected from each farm, totaling 50 samples, processed by the WILLIS-MOLLAY method for qualitative identification of endoparasites. All 25 samples (100\%) from G1 were positive, making it possible to identify parasites of the genera Echinuria spp., Trichostrongylus spp., Amidostomum spp., Heterakis gallinarum, Choanotaenia infundibulum, and oocysts of Eimeria spp. In the second farm, 15 samples (60\%) had endoparasites, including Ascaris spp, Giardia spp., Trichostrongylus spp., with a higher
\end{abstract}


prevalence of Amidostomum spp. The exposition of this parasitic fauna in quails demonstrates the importance of research in the cotton industry and its potential development.

Keywords: Coturniculture; Parasitosis; Pathology; Biosecurity.

\section{Resumen}

El área algodonera es una de las más en expansión, presentándose como una importante alternativa económica, siendo Brasil el quinto productor mundial. El objetivo fue evaluar el perfil endoparásito que afecta a las codornices de dos fincas de la ciudad de Porto Velho - RO. La investigación se realizó en dos granjas de codorniz ponedora de la ciudad, de agosto a noviembre de 2019. Se recolectaron veinticinco muestras de cada granja, totalizando 50 muestras, procesadas por el método WILLIS-MOLLAY para la identificación cualitativa de endoparásitos. Las 25 muestras (100\%) de G1 fueron positivas, lo que permitió identificar parásitos de los géneros Echinuria spp., Trichostrongylus spp., Amidostomum spp., Heterakis gallinarum, Choanotaenia infundibulum y ooquistes de Eimeria spp. En la segunda finca, 15 muestras (60\%) presentaron endoparásitos, incluyendo Ascaris spp, Giardia spp., Trichostrongylus spp., Con mayor prevalencia de Amidostomum spp. La exposición de esta fauna parásita en codornices demuestra la importancia de la investigación en la industria algodonera y su potencial Desarrollo.

Palabras clave: Coturnicultura; Parasitosis; Patología; Bioseguridad.

\section{Introdução}

A coturnicultura é setor em constante expansão, apresentando-se como importante alternativa econômica para pequenos e grandes produtores rurais (Carmo, 2015), o Brasil se comporta como o quinto maior produtor mundial de codornas, com aproximadamente 15 milhões de criações (IBGE, 2011).

As codornas oferecem diversas vantagens, como acelerado crescimento, aumento na produção, requerem pouco espaço, além de maturidade sexual precoce, alta rusticidade e baixo investimento, de modo que atrai os pequenos e médios produtores para essa criação (Martins, 2002; Albino \& Barreto, 2003; Lanna, 2012).

A necessidade de um rápido avanço para a produção de carne e ovos nesse setor se deparou com um obstáculo, comum de sistemas de criação em aviários, a infecção parasitária. No Brasil, é pequeno o conhecimento a respeito dos danos causados pelos endoparasitos no setor da avicultura (Vasconcelos, 2000).

Sendo um problema que prejudica a criação e o desempenho destas pequenas aves, acarretando atraso no crescimento, limitando a taxa de conversão alimentar e, aumentando a suscetibilidade a infecções secundárias (Cardozo \& Yamamura, 2004; Rennó et al. 2008), interferindo no seu comportamento e desenvolvimento reprodutivo, devido a má nutrição e estresse (Freitas et al. 2002). Ocasionando grandes prejuízos econômicos para o setor avícola brasileiro, suscitando ao desequilíbrio econômico e produtivo tanto interno, quanto para exportação (Melo, 2013).

Há uma grande relevância a respeito das granjas nacionais de aves poedeiras e entre os produtores rurais que exercem a agricultura familiar, tendo como meio de subsistência o comércio dos ovos (Parmar \& Davies, 2007; Barrow \& Freitas-Neto, 2011).

Objetivou-se assim avaliar, de forma quantitativa, a carga parasitaria em codornas com dupla aptidão, postura e abate, criadas em duas granjas com sistema vertical automatizado e convencional de produção, na cidade de Porto Velho - RO.

\section{Material e métodos}

A pesquisa foi realizada em duas granjas de codornas poedeiras na cidade de Porto Velho - RO, nos meses de agosto a novembro de 2019. Formando então dois grandes grupos, o da granja 1 (G1) e os da granja 2 (G2). Os animais estudados foram mantidos em galpões, separadas por gaiolas em pequenos grupos, sendo cada uma dessas gaiolas representando uma amostra, sendo fornecido água e ração ad libitum seguindo a rotina de produção. O material fecal foi coletado na granja utilizando-se folhas de plástico tipo PVC, introduzidos em baixo das gaiolas para realizar a captação delas. Após a deposição das fezes pelas aves no PVC, foram coletadas 25 amostras de cada dos dois criatórios de forma randomizada, totalizando 50 amostras, 
armazenadas em coletores universais, devidamente identificados e encaminhados para um Laboratório de Parasitologia Veterinária.

As amostras fecais foram avaliadas utilizando-se do método de WILLIS-MOLLAY (Flutuação simples, 1921), técnica de concentração de ovos, oocistos e cistos que usa o princípio da flutuação em solução saturada com cloreto de sódio. Foram confeccionadas para cada amostra uma lâmina e observadas em microscópio óptico (10x) para identificação qualitativa dos endoparasitas.

\section{Resultados e discussão}

Dentre as amostras analisadas no G1, foram observadas positivas para a presença de endoparasitas 25/25 das amostras (100\%), sendo identificados parasitas dos gêneros Echinuria spp., Trichostrongylus spp., Amidostomum spp., Heterakis gallinarum, Choanotaenia infundibulum, e oocistos de Coccidea esporulados e não esporulados (Eimeria spp.).

No G2 somente 15/25 das amostras (60\%) apresentaram a presença de endoparasitas, entre eles Ascaris spp, Giardia spp., Trichostrongylus spp., com maior prevalência de Amidostomum spp.

Dentre os parasitas o identificados no G1 e G2, foi demonstrado uma baixa infestação por ovos de Ascaridia spp. em codornas japônica, semelhante ao descrito por Vita et al. (2015) e Teixeira \& Lopes (2002), sendo importante lembrar que o transtorno e o estresse causado pela infestação desse nematódeo oportuniza o surgimento de patologias secundárias, como a cólera aviária (Berchieri \& Macari, 2000; Back, 2002; Nunes et al. 2008).

Dispõe como exemplar que contamina a maior parte das aves domésticas o Ascaridia spp., sendo achado maiormente em países subtropicais ou tropicais (Back, 2002; Dehlawi, 2007; Gbif, 2012).

Em aves domésticas, foram identificados por Gomes et al. (2009), Fernandes et al. (2004), Sobral (2010) e Lima et al. (2011), Carneiro (2001), Rosa et al. (2017), Giovannoni e Kubiak (2001), parasitas dos gêneros Ascaridia spp., Eimeria spp., Heterakis spp., como no presente estudo.

Estando presentes em aves silvestres, como demonstrado por Freitas et al. (2002), Kajerova \& Barus (2005), Santos \& Oliveira (2007), Barton et al. (2003) e Carneiro et al. (2011), ao consideraram os supracitados como sendo maior prevalência nessas aves.

Os nematóides Trichostrogylus spp. e Ascaris spp. e a Giardia spp. (protozoário) identificados neste estudo estão entre os endoparasitas mais frequentes em aves exóticas que vivem em cativeiro (Freitas et al. 2002). São comumente encontrados no trato gastrointestinal principalmente de aves Psittaciformes e Columbiformes, sendo agravado naquelas em que aprestam algum grau de imunodeficiência (Freitas et al. 2002; Marques et al. 2007). Brandão et al. (2020) observou Ascaridia sp. e utilizou em seu estudo um novo tipo de tratamento para este nematódeo, utilizando os extratos aquosos de romã e flor de seda.

O gênero Heterakis gallinarum é amplamente distribuído entre as aves, tanto silvestres quanto domésticas, com preferência em parasitar o ceco intestinal, provocando a Heterakiose (Revolledo \& Ferreira, 2009), porém não possui tanto significado patogênico quando não acompanhada de outro endoparasita. Santos et al. (2008) relata que nos últimos anos tornouse mais notórios casos de morte súbita por este patógeno.

A identificação de ovos do nematódeo Amidostomum spp. em amostras fecais de granjas de em S. risoria e Columba livia, também foi mencionado por Lima et al. (2017) na região amazônica. Este parasita foi identificado em cativeiros de aves selvagens que vivem na Colômbia (Corredor et al. 2013) e na Europa, há relatados constantes em patos selvagens (Kavetska et al. 2011).

Foram listadas várias infecções por Choanotaenia infundibulum em galinhas (Gallus Domesticus) nas diferentes regiões do estado de São Paulo, a primeira por Sawada (1970), a partir deste houveram mais relatos do cestódeo em outros grupos de aves (Monte et al. 2018). A infecção por $C$. infundibulum é normalmente assintomática e moderadamente patogênica 
(Premaalatha et al. 2014). Há também um relato feito por Monte et al. (2018), onde se observou a presença de Blastocystis homis em codornas.

A coccidiose é de grande relevância na avicultura pelos danos causados a granja, provocando nas aves além de enterite e diarreia, suscitando em uma grande diminuição na absorção de nutrientes fornecidos na alimentação. Além disso, a patologia propicia que doenças secundárias acometam os animais, tornando-se ainda mais grave (Penha, 2008).

Atinge o epitélio intestinal dos vários grupos de aves, representado por lesões deixadas na área de fixação do parasita (Soave, 2011; Vita et al. 2019). Há quatro espécies de Eimeria que podem ser identificadas em codornas (Teixeira et al., 2004; Mohammad, 2012), baseadas na especificidade do local de desenvolvimento, entre elas: E. tsunodai, com desenvolvimento quase exclusivo no ceco (Tsutsumi, 1972), E. uzura (Tsunoda \& Muraki, 1971) e E. bateri (Bhatia, Pandey \& Pande, 1965) que habitam preferencialmente o duodeno e jejuno, respectivamente (Teixeira et al. 2004, Gesek et al. 2014). A presença de oocistos do gênero Eimeria foi visualizada, como no estudo relatado, em C. japonica em Manaus, Brasil (Monte et al. 2018).

As infecções por helmintos e protozoários revelam uma séria ameaça à saúde de aves exóticas comercialmente criadas, especialmente quando apresentam grandes cargas parasitárias e sinais clínicos aparentes, como diarreia, perda de peso, apatia, e morte (Vicente et al. 1995; Marietto-Gonçalves et al. 2009; Amaral \& Otutumi, 2013).

A introdução e transmissão de parasitas gastrointestinais em aves é possibilitada por um manejo sanitário inadequado, ausência de protocolos de diagnósticos periódicos e consequente tratamento, alta densidade populacional nas gaiolas, água e alimentos contaminados (Urquhart et al. 1990; Marietto-Gonçalves et al. 2009).

De acordo com Silva \& Zocche (2009), são necessárias medidas de biosseguridade nestas granjas e medidas sistemáticas de everminação, deste modo reduziriam a presença de endoparasitas nas criações de aves poedeiras garantindo assim a saúde dessas aves e o bem estar animal, como também assegurando que os produtos e subprodutos se mantenha inócuos e seguros para o consumo humano. A exposição desta fauna parasitária em codornas demonstra a importância das pesquisas na coturnicultura e seu potencial desenvolvimento

\section{Conclusão}

Conclui-se assim que foram encontrados no G1 e G2 os seguintes agentes parasitários Amidostomum spp. Ascaris spp., Choanotaenia infundibulum, Eimeria spp. Echinuria spp., Giardia spp., Heterakis gallinarum Trichostrongylus spp. Vê-se que é de suma importância continuar as pesquisas sobre parasitos em codornas japonesas, afim de melhorar o desempenho e a qualidade destes produtos, aos quais são destinados para consumo humano.

\section{Referências}

Albino, L. F. T. \& Barreto, S. L. T. (2003). Criação de codornas para produção de ovos e carne. Viçosa: Aprenda Fácil. 268 p.

Amaral P.F.G.P. \& Otutumi, L. K. (2013). Prevalência de coccidiose em frangos de corte na integração avícola na Região Noroeste do Estado do Paraná, Brasil. Enc Biosf. 9(16): 1759-1768.

Anual de iniciação científica, 9., (2011). Parapuapebas. Anais... Parapuapebas: Universidade Federal Rural da Amazônia. R072.

Back, A. (2002). Manual de doenças de aves. 1. ed. Cascavel: Unioeste Editora e Gráfica Universitária, 245 p.

Barrow P. A. \& Freitas Neto O. (2011). C. pullorum disease and fowl typhoid, new thoughts on old diseases: a review. Avian Pathol. 40(1): 1-13. http://dx.doi.org/10.1080/03079457.2010.542575. PMid:21331943.

Barton, C. E., Phalen, D. N. \& Snowden, K. F. (2003). Prevalence of microsporidian spores shed by asymptomatic lovebirds: Evidence for a potential emerging zoonosis. Journal of Avian Medicine and Surgery, 17(4): 197-202.

Brandão, P. A. et al. (2020). Effectiveness of flor-de-seda and pomegranate aqueous extracts on eggs of the Heterakoidea Superfamily isolated from naturally infected japanese quais. Rev. Bras. Saúde Prod. Anim., Salvador, 21: 01 - 08, e2121342020. 
Cardozo, S. P. \& Yamamura, M. H. (2004). Parasitas em produção de frangos no sistema de criação tipo colonial/caipira no Brasil. Semina: Ciências Agrárias, 25(1): 63-74.

Carmo, $\quad$ A. $\quad$ K. $\quad$ S. $\quad$ (2015). Sua criação de codornas de forma prática. Encontrado em: < https://www.almanaquedocampo.com.br/imagens/files/Criar\%20codornas.pdf $>$.

Carneiro, V. S. (2001). Composição e estrutura da comunidade de helmintos parasitos de galinhas, Gallus domesticus (L.), no Município de Seropédica, Estado do Rio de Janeiro. 69f. Tese (Mestrado em Parasitologia Veterinária) - Universidade Federal Rural do Rio de Janeiro, Seropédica.

Corredor D. J. G. et al. (2013). Identificação de parasitas gastrointestinais em aves silvestres em cautela. Rev Cie Univer del Zulia, 23(3): 254-258.

Dehlawi, M. S. (2007). The Occurrence of Nematodes in the Intestine of Local (Baladi) Chicken (Gallus gallus domesticus) in Jeddah Province - Saudi Arabia. Scientific Journal of King Faisal University (Basic and Applied Sciences), 8(2): 61-71.

Fernandes, R. M. et al. (2004). Ausência da atividade anti-helmíntica de plantas em frangos de corte naturalmente infectados com Heterakis gallinarum (Schranck, 1788) Madsen, 1949. Ciência Rural, 34(5): 1629-1632.

Freitas, M. F. L. et al. (2002). Parasitos gastrointestinales de aves silvestres em cativeiro em el estado de Pernambuco, Brasil. Parasitologia Latinoamericana, 57(1-2)50-54.

GBIF. (2012). Global Biodiversity Information Facility.

Gesek M. et al. (2014). Alterações patomorfológicas no sistema alimentar de codornas japonesas naturalmente infectadas com Eimeria tsunodai. Touro. Veterinario. Inst. Pulawy 58(1): 41-45. http://dx.doi.org/10.2478/bvip-2014-0007.

Giovannoni, M. \& Kubiak, G. V. L. (2001). Fauna parasitológica paranaense. IV. Lista prévia da ocorrência de helmintos em animais domésticos. Brazilian Archives of Biology and Tecnology, 2(4): 289-292.

Gomes, F. F. et al. (2009). Principais parasitos intestinais diagnosticados em galinhas domésticas criadas em regime extensivo na municipalidade de Campos dos Goytacazes, RJ. Ciência Animal Brasileira, 10(3): 818-822.

IBGE. (2011). Produção da pecuária municipal. Rio de Janeiro: Ministério do Planejamento, Orçamento e Gestão, Instituto Brasileiro de Geografia e Estatística, $63 \mathrm{p}$.

Kajerova, V. \& Barus, V. (2005). Psittacine birds (Aves: Psittaciformes) as new hosts of Baruscapillaria obsignata (Nematoda: Capillariidae). Acta Veterinaria Brno, 74(4): 571-574.

Kavetska K. M. et al. (2011). Revisão do complexo de espécies (Lundahl, 1848) (Nematoda: Amidostomatidae). Amidostomum acutumParasitol, 109(1): 105117. PMid: 21234601.

Lanna, L. L. (2012). Morfologia testicular e reserva espermática na codorna japonesa (Coturnix coturnix japônica). Belo Horizonte. 59 f. Tese (Doutorado em Ciência Animal) - Escola de Medicina Veterinária, Universidade Federal de Minas Gerais, Belo Horizonte.

Lima, E. M. et al. (2011). Perfil parasitológico intestinal de frangos caipiras criados em diferentes sistemas de criação. Anais 9o Seminário Anual de Iniciação Científica, Parapuapebas, AM, (Resumo 72).

Lima, V. F. S. et al. (2017). Gastrointestinal parasites of exotic birds living in captivity in the state of Sergipe, Northeastern Brazil. Rev. Bras. Parasitol. Vet., 26(1): 96-99.

Marietto-Gonçalves, G. A. et al. (2009). Prevalência de endoparasitas em amostras fecais de aves silvestres e exóticas examinadas no Laboratório de Ornitopatologia e no laboratório de Enfermidades Parasitárias da FMVZ-UNESP/Botucatu, SP. Ciência Animal Brasileira, 10(1): 349-354.

Martins, E. N. (2002). Perspectivas do melhoramento genético de codornas no Brasil. In: SIMPÓSIO INTERNACIONAL DE COTURNICULTURA, I., 2002, Lavras. Anais. Lavras: Universidade Federal de Lavras, p. 109-112.

Marques, S. M. T. et al. (2007). Parasitas de pombos (Columba livia) em áreas urbanas de Lages, sul do Brasil. Parasit Latinoam, 62(3-4): 183-187.

Melo, J. K. A. et al. (2013). Potencialidade e extensão da coturnicultura no município de São Bento do Una, Pernambuco. In: XII Jornada de Ensino, Pesquisa e Extensão da UFRPE, 2013. Recife. Anais... Recife.

Mohammad, N. H. (2012). Um estudo sobre o patológico e diagnóstico da infecção por espécies de Eimeria em codornas japonesas. Bras. J. Vet. Res. 1(11): 318-333.

Monte, G. L. S. et al. (2018). Parasitic profiling of Japanese quails (Coturnix japonica) on two farms with conventional production system in the Amazon region. Pesq. Vet. Bras. 38(5): 847-851.

Parmar, D. \& Davies, R. (2007). Fowl typhoid in a small backyard laying flock. Vet. Rec. 160(10):348.

Penha, G. A. (2008) Coccidiose aviária. Revista Cientifica Eletrônica de Medicina Veterinária, 6(11).

Premaalatha, B. et al. (2014). Cestodo intestinal Choanotaenia infundibulum em frango. J. Vet da Malásia. Res. 5(2): 73-75.

Rennó, P. P. et al. (2008). Endoparasitose em aves - revisão de literatura. Revista Científica Eletrônica de Medicina Veterinária, 6(11): 1-6.

Revolledo, L. \& Ferreira, A. J. P. (2009). Patologia Aviária. Manole: São Paulo. 
Research, Society and Development, v. 10, n. 9, e35010917967, 2021

(CC BY 4.0) | ISSN 2525-3409 | DOI: http://dx.doi.org/10.33448/rsd-v10i9.17967

Rosa, M. S. S. et al. (2017). Enteroparasites of the families Eimeriidae and Ascarididae in japanese quail (Coturnix japonica) in the metropolitan region of the Cuiabá River Valley, State of Mato Grosso, Brazil. Veterinária Notícias, 23(2).

Santos, M. G. \& Oliveira, R. C. (2007). Endoparasitos de aves silvestres mantidas em cativeiro. 7f. Trabalho de Conclusão de Curso (Graduação em Ciências Biológicas) - Faculdade Assis Gurgacz, Cascavel.

Santos, G. G. C. et al. (2008). Doenças de aves silvestres diagnosticadas na Universidade Federal do Paraná, Brasil (2003-2007). Pesq Vet Bras. 28(11): 565570 .

Sawada, I. (1970). No cestóide de aves, Choanotaenia infundibulum do Brasil. Touro. Nara Univ. Educ. 19(2): 81-82.

Silva, G. S. \& Zoche, A. T. (2009). Endoparasitoses em aves de produção industrial In: Berchieri Júnior A, et al. ed. Doença das aves. $2^{\mathrm{a}}$ ed. Campinas: FACTA.

Soave. G. L. (2011). Anticoccidianos em rações. Nutritime Revista Eletrônica, 8(1): 1401-1417.

Sobral, F. E. S. (2010). Eficácia anti-helmíntica da Operculina hamiltonii (G. Don) D. F. Austin \& Staples (1983) e Cucurbita pepo L. sobre helmintos gastrintestinais de galinhas caipiras, Gallus domesticus. 71f. Tese (Mestrado em Zootecnia) - Universidade Federal de Campina Grande, Patos.

Teixeira, M., Teixeira Filho, W. L. \& Lopes, C. W. G. (2004). Coccidiose em codornas japonesas (Coturnix japonica) caracterização de uma infecção que ocorre naturalmente em uma fazenda comercial. Revista Bras. Ciênc. Avic. 6(2): 129-134. http://dx.doi.org/10.1590/S1516-635X2004000200010.

Teixeira, M. Z.; Lopes, C. W. G. (2002). Species of the genus Eimeria (Apicomplexa: Eimeriidae) from Japanese quails (Coturnix japonica) in Brazil and E. fluminensis for the preoccupied E. minima of this quail. Revista Brasileira de Ciências Veterinárias, 9: 53-56.

Tsutsumi, Y. (1972). Eimeria tsunodai sp. nov. (Protozoários: Eimeriidae): um coccídio caecal de codornas japonesas (Coturnix japonica). Jpn.J. Vet. Sci. 34(1): 1-9. http://dx.doi.org/10.1292/jvms1939.34.1. PMid: 5063025.

Urquhart, G. M. et al. (1990). Parasitologia veterinária. Rio de Janeiro: Guanabara Koogan, 306 p.

Vasconcelos, O. I. (2000). Parasitose em aves de produção industrial. In: JUNIOR, A. B.; MACARI, M. (Eds.). Doença das aves. 1 ed., Campinas: FACTA, $423-428 \mathrm{p}$.

Vicente, J. J. et al. (1995). Nematóides do Brasil. Parte IV: Nematóides de Aves. Revista Brasileira de Zoologia, 12(1): 1-273.

Vita, G. F. et al. (2019). Atividade anti-helmíntica de Spigelia anthelmia no controle de parasitos gastrintestinais de Gallus Gallus. Scientia Plena, 15(3). 